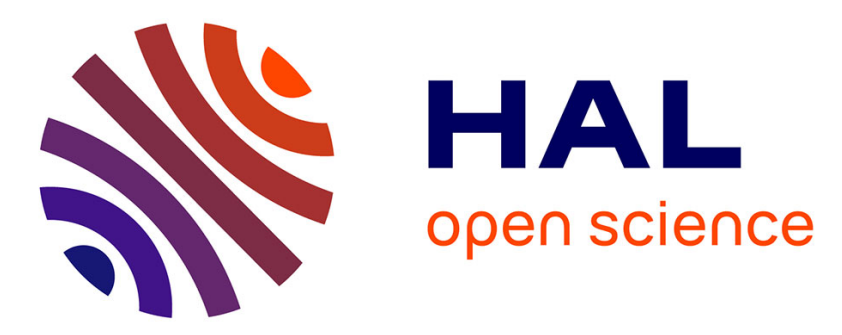

\title{
Bioelectronic sensor mimicking the human neuroendocrine system for the detection of hypothalamic-pituitary-adrenal axis hormones in human blood
}

Seung Hwan Lee, Minju Lee, Heehong Yang, Youngtak Cho, Seunghun Hong, Tai Hyun Park

\section{To cite this version:}

Seung Hwan Lee, Minju Lee, Heehong Yang, Youngtak Cho, Seunghun Hong, et al.. Bioelectronic sensor mimicking the human neuroendocrine system for the detection of hypothalamic-pituitary-adrenal axis hormones in human blood. 2021. hal-03235841

\section{HAL Id: hal-03235841 \\ https://hal.science/hal-03235841}

Preprint submitted on 26 May 2021

HAL is a multi-disciplinary open access archive for the deposit and dissemination of scientific research documents, whether they are published or not. The documents may come from teaching and research institutions in France or abroad, or from public or private research centers.
L'archive ouverte pluridisciplinaire $\mathbf{H A L}$, est destinée au dépôt et à la diffusion de documents scientifiques de niveau recherche, publiés ou non, émanant des établissements d'enseignement et de recherche français ou étrangers, des laboratoires publics ou privés. 


\section{human blood}

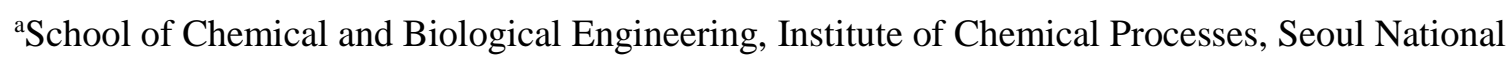
University, Seoul 08826, Republic of Korea

${ }^{\mathrm{b}}$ Department of Bionano Engineering and Bionanotechnology, Hanyang University, Ansan 15588, Republic of Korea

${ }^{\mathrm{c}}$ Department of Physics and Astronomy, Seoul National University, Seoul 08826, Republic of Korea ${ }^{\mathrm{d} P r o t e i n}$ Engineering Laboratory, Discovery Unit, MOGAM Institute for Biomedical Research, Yongin 16924, Republic of Korea

†These authors contributed equally to this work.

*Corresponding author at: Department of Physics and Astronomy, Seoul National University, Seoul 08826, Republic of Korea. Tel.: +82-2-880-1343; Fax: +82-2-884-3002; E-mail: seunghun@snu.ac.kr

*Corresponding author at: School of Chemical and Biological Engineering, Institute of Chemical Processes, Seoul National University, Seoul 08826, Republic of Korea. Tel: +82-2880-8020; Fax: +82-2-875-9348; E-mail: thpark@ snu.ac.kr 


\section{Abstract}

25 In the neuroendocrine system, corticotropin-releasing hormone (CRH) and adrenocorticotropic hormone $(\mathrm{ACTH})$ play important roles in the regulation of the hypothalamic-pituitary-adrenal (HPA) system. Disorders of the HPA system lead to physiological problems, such as Addison's disease and Cushing's syndrome. Therefore, detection of CRH and ACTH is essential for diagnosing disorders related to the HPA system. Herein, receptors of the HPA axis were used to construct a bioelectronic sensor system for the detection of CRH and ACTH. The CRH receptor, corticotropin-releasing hormone receptor 1 (CRHR1), and the ACTH receptor, melanocortin 2 receptor (MC2R), were produced using an Escherichia coli expression system, and were reconstituted using nanodisc (ND) technology. The receptor-embedded NDs were immobilized on a floating electrode of a carbon nanotube field-effect transistor (CNT-FET). The constructed sensors sensitively detected CRH and ACTH to a concentration of $1 \mathrm{fM}$ with high selectivity in real time. Furthermore, the reliable detection of CRH and ACTH in human plasma by the developed sensors demonstrated their potential in clinical and practical applications. These results indicate that CRHR1 and MC2R-based bioelectronic sensors can be applied for rapid and efficient detection of CRH and ACTH.

40

Keywords: bioelectronic sensor; field-effect transistor; nanodisc; hypothalamic-pituitaryadrenal axis; corticotropin-releasing hormone; adrenocorticotropic hormone 


\section{Introduction}

In the human body, the endocrine and nervous systems are used for communication between internal systems (Hiller-Sturmhofel and Bartke 1998). The neuroendocrine system enables communication between the endocrine and nervous systems, and contributes to maintaining homeostasis (Vigas and Jezova 1996). The system consists of the hypothalamus, pituitary gland, and other glands, and can be classified into the hypothalamic-pituitary-adrenal (HPA) axis, hypothalamic-pituitary-thyroid axis (HPT), and hypothalamic-pituitary-gonadal (HPG) axis, depending on the gland (Marques-Deak et al. 2005).

Among them, the HPA axis plays an important role in the regulation of homeostasis in response to stress (Herman and Cullinan 1997). In response to a stressful event, corticotropinreleasing hormone $(\mathrm{CRH})$ is synthesized, and released from the hypothalamus. Subsequently, CRH stimulates corticotropin-releasing hormone receptor 1 (CRHR1) on corticotropic cells in the pituitary, and induces the secretion of adrenocorticotropic hormone (ACTH) from the anterior pituitary. ACTH travels and binds to melanocortin 2 receptor (MC2R) on adrenocortical cells of the adrenal cortex. As a result, glucocorticoid hormone is released from the adrenal cortex (Nieuwenhuizen and Rutters 2008). The secretion of glucocorticoid hormone can be controlled by a negative feedback mechanism in the hypothalamus and pituitary (Juruena et al. 2004).

The HPA axis is associated with such functions of the body as energy usage, digestion, reproduction, and the immune system (Habib et al. 2001; Sapolsky et al. 2000; Smith and Vale 2006). Dysfunction of the HPA system causes pathologic, psychological, and physiological problems, such as Addison's disease, Cushing's syndrome, general adaptation syndrome, posttraumatic stress disorder, obsessive-compulsive disorder, anxiety disorders, and diabetes (Arnett et al. 2016; Coryell et al. 1989; Faravelli et al. 2012; Fries et al. 2015; Kinlein et al. 
2015; Naughton et al. 2014). Because these diseases and syndromes are caused by tumors or damage to the hypothalamus or pituitary, and are subdivided depending on the location of the tumor and impaired part, monitoring of $\mathrm{CRH}$ and $\mathrm{ACTH}$ levels is crucial for making a correct diagnosis and prescribing the correct medication (Hiroi et al. 2001; Pyter et al. 2009). Additionally, CRH and ACTH are prohibited by the World Anti-Doping Agency (WADA), to prevent its misuse (Knoop et al. 2017; Thevis et al. 2006). Therefore, a novel detection system is needed to enforce anti-doping regulations (Duran et al. 2019; Knoop et al. 2017). Furthermore, the CRH- and ACTH-based interaction between the hypothalamus, pituitary, and adrenal cortex is complex and dynamic (Markovic et al. 2011). Therefore, a sensitive and selective sensor system to enable the real-time monitoring of CRH and ACTH is necessary.

Various detection methods have been used to detect CRH and ACTH (Crosby et al. 1988; Håkanson and Sundler 1971; Kertesz et al. 1998; Knoop et al. 2017; Shi et al. 2019). These include radioimmunoassay (RIAs), enzyme-linked immunosorbent assay (ELISA), high-performance liquid chromatography (HPLC), and liquid chromatography-tandem mass spectrometry (LC-MS/MS). Although these methods have shown precise and quantitative results, they require a complicated pretreatment of the sample, long analysis time, large and expensive instruments, and complicated operations (Lee et al. 2015b; Ojeda et al. 2013). To overcome these limitations, electrochemical sensors were introduced (Duran et al. 2019; Li et al. 2017; Moreno-Guzmán et al. 2012; Moreno-Guzmán et al. 2012). However, these sensors require either an antibody $(\mathrm{Ab})$ or a signal amplification for the detection of $\mathrm{CRH}$ and ACTH. These requirements are disadvantageous because they make the sensing process timeconsuming and costly. Therefore, a novel, sensitive, selective, and efficient system is required. Recently, several highly sensitive and selective sensor systems have been reported for the detection of target molecules using the combination of G-protein-coupled receptors 
(GPCRs) with a carbon nanotube field-effect transistor (CNT-FET) (Kim et al. 2013; Kim et al. 2009; Kim et al. 2011). GPCRs contribute to the regulation of physiological functions in the hormonal, neuronal, and sensory systems by interacting with their ligands (Premont and Gainetdinov 2007; Wettschureck and Offermanns 2005). Their specific characteristics of recognizing a corresponding ligand, such as $\mathrm{CRH}$ and $\mathrm{ACTH}$, can be utilized for the construction of a bioelectronic sensor system (Ko and Park 2006; Kwon et al. 2018; Lim et al. 2013; Park et al. 2012). CRHR1 and MC2R are members of the GPCRs, and show high affinity with CRH and ACTH, respectively (Hofmann et al. 1974; Lovejoy et al. 2014). Although the recognition characteristics of CRHR1 and MC2R, for CRH and ACTH have been studied in

Herein, we report a bioelectronic sensor system that mimics the human neuroendocrine HPA axis-related hormones. human physiology, the application of these receptors for the detection of CRH and ACTH through construction of bioelectronic sensor system has not yet been reported.

system for the detection of CRH and ACTH. The receptors that recognize CRH and ACTH, CRHR1 and MC2R, were expressed in Escherichia coli (E. coli), and embedded into nanodiscs (NDs) for reconstitution. To construct the bioelectronic sensor system, the NDs containing each receptor were immobilized on a floating electrode that was combined with a CNT-FET sensor. This sensor system was used to detect the levels of CRH and ACTH in the buffer solution and in human blood plasma. These approaches provide an effective strategy for the detection of

\section{Materials and methods}

\subsection{Materials}

CRHR1 and MC2R cDNA were purchased from Origene (USA), and the MSP1E3D1 bacterial expression vector (pMSP1E3D1) was purchased from Addgene (USA). 
Palmitoyloleoylphosphatidylcholine (POPC) and palmitoyloleoylphosphatidylglycerol (POPG) were purchased from Avanti Polar Lipids (USA). CRH, ACTH, dopamine (DA), epinephrine (EP), serotonin (SE), and human plasma were purchased from Sigma Aldrich (USA).

\subsection{Construction of CRHR1 or MC2R-embedded NDs (CRHRINDs or MC2RNDs)}

Cloning, expression, and purification of CRHR1 and MC2R are included in S.1 of Supplementary Materials. The purified CRHR1 and MC2R were dialyzed against HEPES buffer (20 mM HEPES-NaOH, $100 \mathrm{mM} \mathrm{NaCl}, 25 \mathrm{mM}$ cholate, and $1 \mathrm{mM}$ EDTA, pH 8.0). A lipid mixture (POPC, POPG) was added to the purified CRHR1 and MC2R. After the lipid/receptor mixture was incubated on ice for $20 \mathrm{~min}$, purified and desalted MSP1E3D1 was added (Detailed processes are described in S.1 of Supplementary Materials). The mixed solutions were incubated under gentle stirring for $2 \mathrm{~h}$ at $4{ }^{\circ} \mathrm{C}$. The final mixture contained 1 $\mu \mathrm{M}$ receptors (CRHR1 or MC2R), $8 \mathrm{mM}$ lipids, $25 \mathrm{mM}$ detergents, and $100 \mu \mathrm{M}$ MSP1E3D1. Bio-Beads (Bio-Rad, USA) were added to adsorb the detergent, and the mixtures were incubated with agitation overnight. Afterwards, the mixture was subjected to size exclusion chromatography (SEC) (Superdex 200 Increase 10/300 GL, GE Healthcare, USA) to remove unbound units. Finally, the receptor-embedded NDs were collected, and stored at $4{ }^{\circ} \mathrm{C}$. The quantification of proteins was performed by BCA assay kit (Pierce, IL, USA). The formation of the NDs was observed by SUPRA 55VP field emission scanning electron microscopy (FESEM) (Carl Zeiss, Germany).

\subsection{Fabrication of CNT-FETs with floating electrodes}

Carbon nanotube-field effect transistors (CNT-FET) were fabricated following a previously reported method (Kim et al. 2012; Lee et al. 2015a). Briefly, a CNT dispersion with a 
concentration of $0.05 \mathrm{mg} / \mathrm{mL}$ in 1,2-dichlorobenzene was prepared using sonication for $5 \mathrm{~h}$.

140 For selective CNT assembly, non-polar (octadecyltrichlorosilane, OTS) and polar $\left(\mathrm{SiO}_{2}\right)$ monolayers were patterned on $\mathrm{SiO}_{2}$ substrate using a photolithography process. CNTs were selectively adsorbed on $\mathrm{SiO}_{2}$ regions by immersing the substrate in the CNT solution. Afterwards, source, drain, and floating electrodes $(\mathrm{Pd} / \mathrm{Au} 10 \mathrm{~nm} / 15 \mathrm{~nm})$ were fabricated using photolithography and thermal metal deposition. The length and width of the CNT channels were $170 \mu \mathrm{m}$ and $5 \mu \mathrm{m}$, respectively. The length and width of the floating electrode were 10 $\mu \mathrm{m}$ and $200 \mu \mathrm{m}$, respectively. Finally, a passivation layer (photoresist, DNR) was fabricated to expose only channel regions and to eliminate leakage currents in liquid environments.

\subsection{Functionalization of gold surfaces with NDs containing hormone receptor proteins}

The gold surfaces of floating electrodes were functionalized with hormone receptor proteins following a previously reported method (Yang et al. 2017). V5 antibodies (Thermo scientific) were reduced using 2-mercaptoethylamine hydrochloride (2-MEA) for $1 \mathrm{~h}$ at $37^{\circ} \mathrm{C}$, to prepare half-V5 antibody fragments (Karyakin et al. 2000). By treating the channel regions with solution containing half-V5 antibody fragments at $37{ }^{\circ} \mathrm{C}$ for $1 \mathrm{~h}$, the half-V5 $\mathrm{Ab}$ fragments were immobilized selectively on the gold surface of the floating electrodes. The CRHR1- or MC2RNDs were immobilized on the floating electrodes by the interaction between the V5 epitope of the NDs, and the antigen binding site of the half-V5 antibody.

\subsection{Preparation of samples}

All the tested samples, including CRH, ACTH, DA, SE, and EP, were dissolved in HEPES buffer containing $20 \mathrm{mM}$ HEPES-NaOH, $100 \mathrm{mM} \mathrm{NaCl}$, and $1 \mathrm{mM}$ EDTA, pH 8.0. For the preparation of blood plasma samples, blood plasma was centrifuged at 3,000 $\times g$ for $15 \mathrm{~min}$ at 
$4{ }^{\circ} \mathrm{C}$. The supernatant was stored at $-80{ }^{\circ} \mathrm{C}$, until used. For the electrical measurements, the frozen samples were thawed and mixed with $\mathrm{CRH}$ and $\mathrm{ACTH}$, to prepare blood plasma samples with various concentrations of $\mathrm{CRH}$ and $\mathrm{ACTH}$.

\subsection{Real-time electrical measurements}

Real-time measurement using bioelectronic sensors based on CNT-FETs was performed (Lee et al. 2011; Lee et al. 2015a). A bioelectronic sensor was connected to a semiconductor analyzer (4200-SCS, Keithley, USA). A source-drain voltage of $0.1 \mathrm{~V}$ was constantly applied during electrical measurement, and source-drain currents were monitored. HEPES buffer solution was placed on the channel region of a bioelectronic sensor. Afterwards, various solutions were consecutively introduced into the channel, and the responses of the bioelectronic sensor were monitored.

\section{Results and discussion}

\subsection{Construction of the bioelectronic sensor system}

Fig. 1 shows the HPA axis of the human neuroendocrine system and the bioelectronic sensor system mimicking the human HPA axis for the detection of CRH and ACTH. In the human HPA axis, CRH is secreted from the hypothalamus, and binds to CRHR1 in the pituitary. Subsequently, the pituitary releases ACTH, which interacts with MC2R in the adrenal cortex. CRHR1 and MC2R are receptors in the HPA axis and recognize CRH and ACTH, respectively. To apply these characteristics to a sensor system, CRHR1 and MC2R were produced in an $E$. coli system and embedded in NDs through a reconstitution process.

The CRHR1NDs and MC2RNDs were immobilized on the floating electrode-based CNT-FET sensor system as described previously (Lee et al. 2018; Yang et al. 2017). Based on 
these processes, the bioelectronic sensor system mimicking HPA axis was constructed. In this system, CRHR1NDs and MC2RNDs were used as recognition elements and the floating electrode-based CNT-FET was used as a sensor transducer to detect CRH and ACTH.

Because GPCRs have a transmembrane domain and hydrophobic character with complicated charge distribution (Gafvelin and von Heijne 1994; Grisshammer and Tateu 1995), reconstitution is required to preserve the structure and functions of the GPCR. CRHR1 and MC2R are members of the GPCR family, and reconstitution processes can provide the structural and functional preservation of CRHR1 and MC2R (Bender et al. 2015; Roy et al. 2011). This means that reconstituted CRHR1 and MC2R can recognize the CRH and ACTH selectively and sensitively. NDs have been proposed as a reconstitution method to mimic the native GPCR structure (Denisov and Sligar 2016; Serebryany et al. 2012). In this method, the receptor and lipid bilayer are wrapped with membrane scaffold proteins (MSPs). The stability of NDs embedded with GPCRs and the superiority in detection by the sensor is reported (Oh et al. 2019). However, studies describing the reconstitutions of CRHR1 and MC2R using NDs or the application of the CRHR1ND and MC2RND in the sensor have not been carried out before.

The sensor transducer was also improved by the addition of floating electrodes to the CNT-FET (Kim et al. 2012; Lee et al. 2012; Lee et al. 2015a). The combination of the improved sensor and reconstituted receptors resulted in enhanced performance in terms of sensitivity and selectivity as previously described (Lee et al. 2018; Yang et al. 2017). In previous studies, floating electrode CNT-FET has shown high sensitivity in detecting analytes and high selectivity was achieved by immobilization of reconstituted receptors (Lee et al. 2018; Yang et al. 2017). Because CRHR1 and MC2R can recognize CRH and ACTH in the human HPA axis with high sensitivity and selectivity, CRHR1NDs and MC2RNDs play critical roles in 
enhancing the sensing performances by combining with floating electrode CNT-FET.

212

3.2 Characterization of CRHR1 and MC2R expressed in Human embryonic kidney (HEK-293) cells and E. coli

Membrane proteins, especially transmembrane proteins such as GPCRs, have been expressed in HEK-293 cells, and their functions have been tested in many studies (Atwood et al. 2011; Liberles and Buck 2006). HEK-293 cells were transfected with the gene for CRHR1 or MC2R, and their ligand binding function was evaluated using a Dual-Glo luciferase assay system (see the details in the S.1 of Supplementary Materials). In Figs. 2a and c, CRHR1- and MC2Rthat both CRHR1 and MC2R were successfully expressed in HEK-293 cells, and retained their ligand-binding interactions.

CRHR1 or MC2R were stimulated with $1 \mu \mathrm{M}$ of (CRH, ACTH, DA, EP, and SE). DA, EP, and SE are neurotransmitters that contribute to the control of body functions as chemical results indicate that CRHR1 and MC2R can discriminate their ligand. 
solubilized, and purified using $\mathrm{Ni}^{2+}$ affinity chromatography. For the formation of NDs, the MSP1E3D1 was also successfully overexpressed in E. coli, and purified (S.1 of Supplementary Materials). Fig. 2e shows the results of the western blot analysis of the produced CRHR1 (left), and MC2R (right) (See the detailed procedures in S.1 of Supplementary Materials). The revealed bands correctly indicate the estimated size of the receptors, which implies that CRHR1 and MC2R were successfully expressed in E. coli. The receptor-embedded NDs were constructed by mixing the receptor, lipids, and MSP1E3D1. Fig. 2f presents a SEM image of CRHR1NDs, showing that the produced NDs have the appropriate discoidal shape, and that their average size was approximately $20 \mathrm{~nm}$. These results indicate that the production, purification, and reconstitution of CRHR1 and MC2R were successfully achieved. Bacterial expression is a simple, convenient, inexpensive, and efficient for production of recombinant proteins (Park et al. 2016). In this study, E. coli was used as a representative host cell as commercial CRHR1 and MCR2 are expensive and their functionality is not guaranteed. Although E. coli displays robust production of recombinant proteins, the expression and purification of GPCR is difficult because it has complicated structures with a hydrophobic character (Kiefer et al. 1996; Yang et al. 2015). In this regard, overexpression of CRHR1 and MC2R using an E. coli expression system and their purification are a remarkable aspect of this work. Furthermore, the purified CRHR1 and MC2R were reconstituted with the ND technology for the first time. These trials allowed the detection of CRH and ACTH using CRHR1 and MC2R, which had structural stability and in vivo-like functionality.

\subsection{Optical and electrical characterization of the sensor system}

An optical image of a CNT-FET with floating electrodes is shown in Fig. 3a. Detailed fabrication procedures of a floating electrode-based CNT-FET system are described in the 
Materials and Methods section. Floating electrode-based CNT-FET sensors have been reported to exhibit better sensing performance than a conventional CNT-FET sensor system (Lee et al. 2012; Lee et al. 2015a). The size of the sensor $2 \mathrm{~cm} \times 1 \mathrm{~cm}$ and the sensor has two CNT channel regions.

The fabrication of floating electrode CNT-FET system was verified using fieldemission scanning electron microscopy (FE-SEM). The SEM image of the channel region in the fabricated CNT-FET with floating electrode is shown in Fig. 3b. Five floating electrodes were constructed between the source and drain electrodes. The length and width of the floating electrode were about $10 \mu \mathrm{m}$ and $200 \mu \mathrm{m}$, respectively. Furthermore, the right image of Fig. 3b is the enlarged SEM image of one floating electrode, which shows the CNT channel region under the floating electrode. The CNTs were well-aligned between the source and drain electrodes. The width of the CNT channel was about $5 \mu \mathrm{m}$. These results indicate that the CNT channel and floating electrodes of a CNT-FET system were well-fabricated. Especially, the CNTs were selectively attached on $\mathrm{SiO}_{2}$ regions. Fig. 3c shows the I-V curves of a floating electrode-based CNT-FET system before and after the immobilization of NDs. The bias voltage was varied from $-0.4 \mathrm{~V}$ to $+0.4 \mathrm{~V}$ and source-drain currents were monitored using a semiconductor analyzer. This result shows that the immobilization of NDs caused an increase in resistance and the slope of the I-V profile after the immobilization decreased. However, the linear behavior of the I-V curves was maintained in both before and after the immobilization of NDs on floating electrodes. This indicates a stable ohmic behavior despite the immobilization of NDs on the floating electrodes. Fig. 3d shows the liquid gate profiles of a sensor system before and after the immobilization of NDs. Source-drain currents were monitored in the gate bias range from $-0.25 \mathrm{~V}$ to $0.25 \mathrm{~V}$. The currents decreased when the gate voltage increased, which indicates a p-type characteristic. Although the immobilization of NDs 
caused a decrease in channel conductance, the p-type behavior was maintained even after the immobilization of NDs on the floating electrodes. The decrease in channel conductance would be from the negatively-charged C-terminuses of NDs on the floating electrodes (Lee et al. 2018). These results confirm that the gate characteristic of a CNT-FET with floating electrodes was maintained after the immobilization of NDs, which indicates the constructed system could be appropriate for the detection of $\mathrm{CRH}$ and $\mathrm{ACTH}$ as a biosensor.

\subsection{Electrical measurement of CRH and ACTH in a standard sample}

Fig. 4a shows the real-time responses of a bioelectronic sensor functionalized with CRHR1NDs to different concentrations of CRH. The bioelectronic sensor was connected to a semiconductor analyzer, and a source-drain bias voltage of $0.1 \mathrm{~V}$ was constantly applied to the bioelectronic sensor during the measurement. Source-drain currents were monitored while $\mathrm{CRH}$ solutions were introduced to the channel region of the bioelectronic sensor. Here, $\Delta \mathrm{G} \mathrm{G}_{0}$ was used as the signal of the bioelectronic sensor, where $\Delta \mathrm{G}$ and $\mathrm{G}_{0}$ are the conductance change and original conductance of the CNT-FET, respectively (Lee et al. 2015a). The addition of the $\mathrm{CRH}$ solutions in the range of $1 \mathrm{fM}$ to $1 \mathrm{pM}$ led to increase in the conductance of the CRHR1NDs-functionalized bioelectronic sensor. However, a bare CNT-FET did not respond to various concentrations of CRH (Fig. S1a of the Supplementary Materials). This implies that the responses arose from specific binding events between CRHR1 and CRH. Presumably, the binding events induced a structural change in receptor proteins, leading to a change in the charge state of receptor proteins on the floating electrodes. This resulted in a change in the channel conductance of the bioelectronic sensor via Schottky barrier modulation (Kim et al. 2012; Lee et al. 2018). The result shows that the bioelectronic sensor functionalized with CRHR1NDs could sensitively respond to CRH in real-time. 
Fig. $4 \mathbf{b}$ shows the dose-dependent responses of bioelectronic sensors functionalized

with CRHR1NDs to different concentrations of CRH. The responses were obtained by normalizing each sensor signal at a certain concentration with respect to the maximum signal value at the highest concentration. Each data point and error bar represent the average value and standard deviation of experiments using several bioelectronic sensors. The responses to $\mathrm{CRH}$ began to be observable at a concentration of $1 \mathrm{fM}$, and increased as the concentrations of $\mathrm{CRH}$ increased. The curve reached a plateau after the addition of $10 \mu \mathrm{M}$ CRH to the bioelectronic sensor because the binding sites of CRHR1 were occupied by CRH. These responses were analyzed using a Langmuir isotherm-based model as reported previously (Lee et al. 2018; Park et al. 2014; Song et al. 2014). The estimated equilibrium constant between CRHR1 and CRH obtained using bioelectronic sensors was $5.70 \times 10^{9} \mathrm{M}^{-1}$. This value is quite similar to previously reported values (Grammatopoulos et al. 1999; Hatzoglou et al. 1996; McDowell et al. 2012). These results indicate that the bioelectronic sensor responded to CRH dose-dependently and reproducibly.

Fig. 4c shows the real-time responses of a bioelectronic sensor functionalized with CRHR1NDs to various hormones and neurotransmitters at a concentration of $1 \mathrm{nM}$. Sourcedrain currents were monitored while introducing SE, EP, ACTH, and CRH solutions to the channel region of the bioelectronic sensor. The addition of $1 \mathrm{nM} \mathrm{SE}$, EP, and ACTH solutions did not result in conductance changes, while the addition of CRH caused an increase in channel conductance. These results demonstrate that the bioelectronic sensor specifically responded to CRH with high selectivity. In human physiology, SE, EP, CRH and ACTH have critical roles for regulation and homeostasis of body systems through interactions with their counterpart receptors. Because the reconstituted CRHR1NDs have a similar structure and function with those of the receptors in the human body, the CRHR1ND could selectively discriminate CRH 
from other hormones. Accordingly, CRH could cause the conformational change of CRHR1ND similar with that of CRHR1 in vivo, while other hormones could not induce the structural change of CRHR1ND. This alteration could induce the change of electrical charges in the receptor, resulting in increased channel conductance in the sensor system.

Fig. 4d displays the real-time responses of a bioelectronic sensor functionalized with MC2RNDs to ACTH. The real-time measurement was conducted in the same way as in the experiment shown in Fig. 4a. The injection of ACTH solutions in the range of 1 to $100 \mathrm{fM}$ caused a sharp increase in the channel conductance of the bioelectronic sensor. A bare CNTFET did not exhibit conductance changes to various concentrations of ACTH (Fig. S1b of the Supplementary Materials). Note that the electrical responses resulted from the specific binding between MC2R and ACTH. The interaction and binding between MC2R and ACTH induced structural changes in MC2R proteins. The structural changes resulted in a change in the charge state of the proteins on the floating electrodes. This in turn led to altered channel conductance of the bioelectronic sensor via Schottky barrier modulation (Kim et al. 2012; Lee et al. 2018). These results imply that the bioelectronic sensor functionalized with MC2RNDs could detect ACTH with high sensitivity in real-time.

In Figs. 4a and d, we performed the tests in the process from low to high concentrations of target molecules. Because the responses of our sensor system were based on the binding of CRH to the CRHR1 or ACTH to the MC2R, the binding sites of CRHR1 or MC2R could be occupied by CRH or ACTH. Therefore, if a sensitivity test was performed from high to low concentrations, the high concentration of CRH or ACTH would first occupy the binding sites of receptors and would disturb the binding of the low concentration of CRH or ACTH molecules. Hence, we performed the sensitivity test of sensors from low to high concentrations of target molecules. 
Fig. 4e shows the dose-dependent responses of bioelectronic sensors functionalized with MC2RNDs to various concentrations of ACTH. The responses were obtained in the same way as in the experiment shown in Fig. $\mathbf{4 b}$, and were analyzed based on a Langmuir isothermbased model (Lee et al. 2018; Song et al. 2014). The bioelectronic sensors began to exhibit responses to $\mathrm{ACTH}$ at a concentration of $1 \mathrm{fM}$. The responses increased as the concentration of ACTH increased in a dose-dependent manner. The curve was saturated after the injection of $10 \mu \mathrm{M}$ ACTH into the bioelectronic sensor because the binding sites of MC2R were occupied by ACTH. The equilibrium constant between MC2R and ACTH was estimated to be $5.47 \times$ $10^{10} \mathrm{M}^{-1}$. This value is similar to a previously reported value using a cell-based system

Fig. 4f shows the real-time responses of a bioelectronic sensor functionalized with MC2RNDs to various hormones and neurotransmitters at a concentration of $1 \mathrm{nM}$. The introduction of $\mathrm{SE}, \mathrm{EP}$, and $\mathrm{CRH}$ solutions did not induce a conductance change in the bioelectronic sensor, and only the addition of ACTH solution led to significant increase in the channel conductance. These results show that MC2R on the bioelectronic sensor could discriminate ACTH from other hormones or neurotransmitters. As noted earlier, SE, EP, CRH, and ACTH exist in the human blood and play important roles in the regulation and homeostasis of bodily functions. The interaction of these hormones or neurotransmitters with their counterpart receptors results in the regulation of various processes in the body. Based on this, CRHR1NDs and MC2RNDs were reconstituted and combined with a floating electrode-based CNT-FET system. Hence, CRH and ACTH were selectively detected among other hormones or neurotransmitters by utilizing our sensor system (Figs. $\mathbf{4 c}$ and $\mathbf{f}$ ). Therefore, the developed 
detection of CRH and ACTH.

380

3.5 Evaluation of the sensing performance for $C R H$ and ACTH in human blood plasma

To assess the possibility of the bioelectronic sensor for medical and clinical use, the detection of $\mathrm{CRH}$ and $\mathrm{ACTH}$ in human blood plasma was performed. The blood plasma contains many compounds, which could affect the conductance change of sensor platforms (Lim et al. 2014). Thus, plasma sample was serially diluted with buffer solution to $1 / 10^{6}$. The non-specific influence of blood plasma was tested through injection of diluted plasma samples to a bare CNT-FET (Fig. S2 of the Supplementary Materials). The plasma diluted $1 / 10$ to $1 / 10^{2}$ had an effect on the conductance change of the sensor platform (data not shown). The diluted plasma samples in the range of $1 / 10^{3}$ to $1 / 10^{6}$ did not affect the conductance change of the bioelectronic sensor platform. Based on these results, a $1 / 10^{4}$ diluted plasma sample was mixed with CRH or $\mathrm{ACTH}$, and different concentrations of $\mathrm{CRH}$ or ACTH samples from $10^{-18}$ to $10^{-5} \mathrm{M}$ were prepared. Fig. 5 shows the dose-dependent responses of a CRHR1NDs or MC2RNDsfunctionalized bioelectronic sensor to various concentrations of CRH (Fig. 5a) or ACTH (Fig. 5b) in the diluted plasma. The bioelectronic sensors dose-dependently responded to the CRH or ACTH in diluted plasma. The curves reached a plateau after the injection of $10^{-6} \mathrm{M}$ of CRH or ACTH. Typically, CRH and ACTH levels in blood plasma are in the picomolar range (Linton et al. 1987; Talbot et al. 2003). These levels are in the detection range of our bioelectronic sensor, based on the results shown in Fig. 5. When the dose-response curves of CRHR1NDs or MC2RNDs to $\mathrm{CRH}$ or ACTH were compared in plasma environment (Fig. 5) and in the buffer condition (Fig. 4), similar slopes were observed. The equilibrium constant between CRHR1 and CRH in diluted plasma was estimated as $2.20 \times 10^{9} \mathrm{M}^{-1}$, and the equilibrium constant between MC2R and ACTH in diluted plasma was estimated as $1.32 \times 10^{11} \mathrm{M}^{-1}$. The 
equilibrium constants in the plasma environment were similar to those from the experiments under buffer condition shown in Fig. 4b (CRHR1 to CRH) and Fig. 4e (MC2R to ACTH). In order to verify the selectivity of the bioelectronic sensor functionalized with CRHR1NDs and MC2RNDs in human blood plasma, a 1/10 4 diluted plasma sample was mixed with various hormones and neurotransmitters, such as SE, EP, CRH, and ACTH. The intensities of the responses to $\mathrm{CRH}$ and $\mathrm{ACTH}$ were quantitatively compared with those of the other hormones and neurotransmitters of $1 \mathrm{nM}$ concentration. Fig. $\mathbf{5 c}$ shows the selective responses of a CRHR1NDs functionalized bioelectronic sensor to CRH with $1 \mathrm{nM}$. The sensors showed significant changes in conductance in response to $\mathrm{CRH}$. However, other hormones and neurotransmitters such as the SE, EP, and ACTH did not cause changes in the conductance of the sensor. Fig. 5d shows the selective responses of a MC2RNDs functionalized bioelectronic sensor to ACTH with $1 \mathrm{nM}$. The sensors showed a significant response to $1 \mathrm{nM}$ ACTH. However, the introduction of SE, EP, and CRH induced negligible changes in the conductance of the sensor. These results indicate that the biosensors could sensitively and selectively detect $\mathrm{CRH}$ or $\mathrm{ACTH}$ in blood plasma as well as pure environment such as buffer. Our biosensor could be also effectively applied for the diagnosis of HPA axis-related diseases and anti-doping regulations. Therefore, the biosensors developed in this work could have potential for practical and clinical applications.

Although several studies have reported the detection of CRH and ACTH before, our receptor-based system has higher sensitivity and selectivity than the other systems (Table 1). Recently, laser desorption/ionization mass spectrometry (LDI MS)-based analysis has been introduced as an advanced method of MS that features high sensitivity, accuracy, and in-second detection (Sun et al. 2018; Vedarethinam et al. 2019). Nevertheless, it is still difficult to make a conclusive comparison with the other systems because LDI MS-based analysis is yet to be 
applied for the detection of $\mathrm{CRH}$ and ACTH. Although electrochemical methods have shown good sensitivity, most of them required Ab-based detection approaches, and such approaches are time-consuming. Furthermore, several sensors additionally required signal amplification for detection due to their low sensitivity. Therefore, the sensing procedures are less efficient, more expensive, and time-consuming. However, the sensor developed in this study can detect $\mathrm{CRH}$ and $\mathrm{ACTH}$ using receptors like those of the human neuroendocrine system. This development circumvents the $\mathrm{Ab}$ requirement and signal amplification in the detection of $\mathrm{CRH}$ and ACTH. Therefore, our detection system is superior to the other reported systems in terms of cost, analysis time, and efficiency. Furthermore, the receptor-based sensing and recognition approaches developed in this study can be applicable for the detection of target molecules by other label-free sensing and recognition methods (Liu et al. 2020; Sun et al. 2019).

\section{Conclusions}

We developed a bioelectronic sensor system that mimicked the human neuroendocrine system for the detection of the HPA axis hormones, CRH and ACTH. The CRH receptor and ACTH receptor, CRHR1 and MC2R, were produced in E. coli, and reconstituted using NDs. The receptors were combined with a floating electrode-based CNT-FET. The bioelectronic sensor exhibited real-time responses to $\mathrm{CRH}$ and $\mathrm{ACTH}$ at concentrations down to $1 \mathrm{fM}$, and discriminated each hormone from other hormones and neurotransmitters. The performance was maintained in human plasma samples. These results indicate that our system can be applied to basic neuroendocrine-related research, and shows excellent potential for use in medical and practical applications. 
449 Conflict of Interest: The authors declare no competing financial interest.

\section{Author Contributions}

451 ML fabricated bioelectronic sensors and performed sensing measurements. YC characterized 452 the sensors. SHL and HY prepared CRHR1 and MC2R and stabilized in NDs. THP and SH are 453 responsible for the project and contributed to data analyses. The manuscript was written 454 through contributions of all authors. All authors have given approval to the final version of the 455 manuscript.

\section{Acknowledgments}

457 This work was supported by the National Research Foundation of Korea (NRF) funded by the 458 Ministry of Science and ICT (MSIT) of Korea (nos. 2013M3A6B2078961, 2018R1A2B3004498, 459 2018R1C1B5085757), the Ministry of Education (2018R1A6A1A03024231), and the European 460 Research Council (ERC) under the European Union's Horizon 2020 research and innovation 461 program (grant agreement no. 682286). S.H. also acknowledges the support from Samsung 462 Electronics, and from the National Research Foundation of Korea (no. 2017R1A2B2006808). 463 This work was also supported by KIST Institutional Program (no. 2E29530). 
Arnett, M.G., Muglia, L.M., Laryea, G., Muglia, L.J., 2016. Neuropsychopharmacology 41(1), 245-260.

Atwood, B.K., Lopez, J., Wager-Miller, J., Mackie, K., Straiker, A., 2011. BMC Genomics 12(1), 14.

Bender, J., Engeholm, M., Ederer, M.S., Breu, J., Møller, T.C., Michalakis, S., Rasko, T., Wanker, E.E., Biel, M., Martinez, K.L., 2015. PLoS One 10(9), e0136768.

Coryell, W.H., Black, D.W., Kelly, M.W., Noyes Jr, R., 1989. Psychiatry Res. 30(3), 243-251.

Di Chiara, G., Morelli, M., Consolo, S., 1994. Trends Neurosci. 17(6), 228-233.

Duran, B., Castañeda, E., Armijo, F., 2019. Biosens. Bioelectron. 131, 171-177.

477 Fries, G.R., Vasconcelos-Moreno, M.P., Gubert, C., Santos, B.T.M.Q.d., Sartori, J., Eisele, B., Ferrari, P., Fijtman,

Gafvelin, G., von Heijne, G., 1994. Cell 77(3), 401-412.

Grisshammer, R., Tateu, C., 1995. Q. Rev. Biophys. 28(3), 315-422.

Habib, K.E., Gold, P.W., Chrousos, G.P., 2001. Endocrinol. Metab. Clin. North Am. 30(3), 695-728.

Hadjiconstantinou, M., Cohen, J., Neff, N.H., 1983. J. Neurochem. 41(5), 1440-1444.

491 Hofmann, K., Montibeller, J.A., Finn, F.M., 1974. Proc. Natl. Acad. Sci. U.S.A. 71(1), 80-83. 
Kiefer, H., Krieger, J., Olszewski, J.D., von Heijne, G., Prestwich, G.D., Breer, H., 1996. Biochemistry 35(50), 16077-16084.

Kim, B., Lee, J., Namgung, S., Kim, J., Park, J.Y., Lee, M.-S., Hong, S., 2012. Sens. Actuators. B. Chem. 169, 182-187.

Kim, B., Song, H.S., Jin, H.J., Park, E.J., Lee, S.H., Lee, B.Y., Park, T.H., Hong, S., 2013. Nanotechnology 24(28), 285501.

Kim, T.H., Lee, S.H., Lee, J., Song, H.S., Oh, E.H., Park, T.H., Hong, S., 2009. Adv. Mater. 21(1), 91-94.

Kim, T.H., Song, H.S., Jin, H.J., Lee, S.H., Namgung, S., Kim, U.-k., Park, T.H., Hong, S., 2011. Lab. Chip 11(13), 2262-2267.

Kinlein, S.A., Wilson, C.D., Karatsoreos, I.N., 2015. Front. Psychiatry 6(31), 1-9.

Knoop, A., Thomas, A., Bidlingmaier, M., Delahaut, P., Schänzer, W., Thevis, M., 2017. Anal. Methods 9(29), 4304-4310.

Ko, H.J., Park, T.H., 2006. Biol. Chem. 387(1), 59-68.

Kwon, O.S., Song, H.S., Park, T.H., Jang, J., 2018. Chem. Rev. 119(1), 36-93.

Lee, J., Jo, M., Kim, T.H., Ahn, J.-Y., Lee, D.-k., Kim, S., Hong, S., 2011. Lab. Chip 11(1), $52-56$.

Lee, J., Lee, H., Kim, T., Jin, H.J., Shin, J., Shin, Y., Park, S., Khang, Y., Hong, S., 2012. Nanotechnology 23(8), 085204.

Lee, M., Jung, J.W., Kim, D., Ahn, Y.-J., Hong, S., Kwon, H.W., 2015a. ACS nano 9(12), 11728-11736.

Lee, M., Yang, H., Kim, D., Yang, M., Park, T.H., Hong, S., 2018. Sci. Rep. 8(1), 13945.

Lee, S.H., Lim, J.H., Park, J., Hong, S., Park, T.H., 2015b. Biosens. Bioelectron. 71, 179-185.

Li, N., Larin, E., Kerman, K., 2017. Sensors 17(12), 2836.

Liberles, S.D., Buck, L.B., 2006. Nature 442(7103), 645-650.

Lim, J.H., Park, J., Ahn, J.H., Jin, H.J., Hong, S., Park, T.H., 2013. Biosens. Bioelectron. 39(1), 244-249.

Lim, J.H., Park, J., Oh, E.H., Ko, H.J., Hong, S., Park, T.H., 2014. Adv. Healthc. Mater. 3(3), 360-366.

Linton, E., McLean, C., Kruseman, A.N., Tilders, F., Van der Veen, E., Lowry, P., 1987. J. Clin. Endocrinol. Metab. 64(5), 1047-1053.

Liu, W., Sun, S., Huang, Y., Wang, R., Xu, J., Liu, X., Qian, K., 2020. Chem. Asian J. 15(1), 56-60.

Lovejoy, D.A., Chang, B.S., Lovejoy, N.R., del Castillo, J., 2014. J. Mol. Endocrinol. 52(3), T43-T60.

Mariussen, E., Fonnum, F., 2001. Toxicology 159(1-2), 11-21.

Markovic, V.M., Cupic, Z., Vukojevic, V., Kolar-Anic, L., 2011. Endocr. J. 58(10), 889-904.

Marques-Deak, A., Cizza, G., Sternberg, E., 2005. Mol. Psychiatry 10(3), 239-250. 
McDowell, E.N., Kisielewski, A.E., Pike, J.W., Franco, H.L., Yao, H.H., Johnson, K.J., 2012. PLoS One 7(10), e47359.

Melmed, S., 2010. The pituitary, third ed. Academic press, London.

Moreno-Guzmán, M., Ojeda, I., Villalonga, R., González-Cortés, A., Yánez-Sedeno, P., Pingarrón, J.M., 2012. Biosens. Bioelectron. 35(1), 82-86.

Moreno-Guzmán, M., González-Cortés, A., Yáñez-Sedeño, P., Pingarrón, J.M., 2012. Electroanalysis 24(5), 11001108.

Naughton, M., Dinan, T.G., Scott, L.V., 2014. Handb. Clin. Neurol. 124, 69-91.

Nieuwenhuizen, A.G., Rutters, F., 2008. Physiol. Behav. 94(2), 169-177.

Oh, J., Yang, H., Jeong, G.E., Moon, D., Kwon, O.S., Phyo, S., Lee, J., Song, H.S., Park, T.H., Jang, J., 2019. Anal. Chem. 91(19), 12181-12190.

Ojeda, I., Moreno-Guzmán, M., González-Cortés, A., Yáñez-Sedeño, P., Pingarrón, J.M., 2013. Analyst 138(15), 4284-4291.

Park, E.J., Park, J., Song, H.S., Kim, S.J., Jung, K.C., Kim, S.-M., Cho, D.-g., Kim, D., Park, K.S., Hong, S., 2014. Biosens. Bioelectron. 61, 140-146.

Park, J., Lim, J.H., Jin, H.J., Namgung, S., Lee, S.H., Park, T.H., Hong, S., 2012. Analyst 137(14), 3249-3254.

Park, S.J., Lee, S.H., Yang, H., Park, C.S., Lee, C.-S., Kwon, O.S., Park, T.H., Jang, J., 2016. ACS Appl. Mater. Interfaces 8(42), 28897-28903.

Premont, R.T., Gainetdinov, R.R., 2007. Annu. Rev. Physiol. 69, 511-534.

Pyter, L.M., Pineros, V., Galang, J.A., McClintock, M.K., Prendergast, B.J., 2009. Proc. Natl. Acad. Sci. U.S.A. 106(22), 9069-9074.

Roy, S., Roy, S.J., Pinard, S., Taillefer, L.-D., Rached, M., Parent, J.-L., Gallo-Payet, N., 2011. Mol. Endocrinol. 25(11), 1961-1977.

Sapolsky, R.M., Romero, L.M., Munck, A.U., 2000. Endocr. Rev. 21(1), 55-89.

Serebryany, E., Zhu, G.A., Yan, E.C., 2012. Biochim. Biophys. Acta 1818(2), 225-233.

Shi, J., Dhaliwal, P., Zheng, Y.Z., Wong, T., Straseski, J.A., Cervinski, M.A., Shajani-Yi, Z., DeMarco, M.L., 2019. Clin. Chem. 65(11), 1397-1404.

Smith, S.M., Vale, W.W., 2006. Dialogues Clin. Neurosci. 8(4), 383-395.

Song, H.S., Jin, H.J., Ahn, S.R., Kim, D., Lee, S.H., Kim, U.-K., Simons, C.T., Hong, S., Park, T.H., 2014. ACS nano 8(10), 9781-9789.

Sun, S., Wang, R., Huang, Y., Xu, J., Yao, K., Liu, W., Cao, Y., Qian, K., 2019. Small 15(34), 1902441.

Sun, X., Huang, L., Zhang, R., Xu, W., Huang, J., Gurav, D.D., Vedarethinam, V., Chen, R., Lou, J., Wang, Q., 2018. ACS Cent. Sci. 4(2), 223-229.

Talbot, J., Kane, J., White, A., 2003. Ann. Clin. Biochem. 40(5), 453-471. 
560 Thevis, M., Bredehöft, M., Geyer, H., Kamber, M., Delahaut, P., Schänzer, W., 2006. Rapid Commun. Mass 561 Spectrom. 20(23), 3551-3556.

562 Vedarethinam, V., Huang, L., Xu, W., Zhang, R., Gurav, D.D., Sun, X., Yang, J., Chen, R., Qian, K., 2019. Small 563 15(3), 1803051.

564 Vigas, M., Jezova, D., 1996. Bratisl. Lek. Listy 97(2), 63-71.

565 Wettschureck, N., Offermanns, S., 2005. Physiol. Rev. 85(4), 1159-1204.

566 Yang, H., Kim, D., Kim, J., Moon, D., Song, H.S., Lee, M., Hong, S., Park, T.H., 2017. ACS nano 11(12), 1184756711855 .

568 Yang, H., Song, H.S., Ahn, S.R., Park, T.H., 2015. Biotechnol. Bioprocess Eng. 20(3), 423-430.

569

570 
Table 1. Comparison of performance between the developed sensor with other reported sensors.

\begin{tabular}{|c|c|c|c|c|c|}
\hline Analyte & $\begin{array}{c}\text { Recognition } \\
\text { element }\end{array}$ & $\begin{array}{c}\text { Analytical } \\
\text { method }\end{array}$ & $\begin{array}{c}\text { Detection limit } \\
(\mathbf{M})\end{array}$ & $\begin{array}{l}\text { Assay time } \\
\text { (min) }\end{array}$ & Reference \\
\hline \multirow{3}{*}{ CRH } & - & LC-MS/MS & $52.1 \times 10^{-12}$ & $\begin{array}{c}>20 \mathrm{~min} \\
(\text { Precipitation: }>1 \mathrm{hr})\end{array}$ & (Knoop et al. 2017) \\
\hline & Antibody & EIS & $0.7 \times 10^{-6}$ & $>100 \mathrm{~min}$ & (Duran et al. 2019) \\
\hline & Receptor & FET & $10^{-15}$ & Real-time & This study \\
\hline \multirow{4}{*}{ ACTH } & - & LC-MS/MS & $2.0 \times 10^{-12}$ & $\begin{array}{c}>3 \mathrm{~min} \\
(\text { Precipitation: }>1 \mathrm{hr})\end{array}$ & (Shi et al. 2019) \\
\hline & Antibody & Voltammetry & $4.0 \times 10^{-15}$ & $>100 \mathrm{~min}$ & $\begin{array}{c}\text { (Moreno-Guzmán et } \\
\text { al. 2012) }\end{array}$ \\
\hline & Antibody & EIS & $22.0 \times 10^{-15}$ & $>100 \mathrm{~min}$ & (Li et al. 2017) \\
\hline & Receptor & FET & $10^{-15}$ & Real-time & This study \\
\hline
\end{tabular}

Abbreviation. EIS: electrochemical impedance spectroscopy. LC/MS: liquid chromatography 


\section{Figure captions}

577 Fig. 1. Schematic of the HPA axis of the human neuroendocrine system and bioelectronic 578 sensor system. In the human HPA axis, the hypothalamus, pituitary, and adrenal cortex 579 communicate with interactions between CRHR1 or MC2R and CRH or ACTH, respectively. 580 The bioelectronic sensor system mimics this system, in which CRHR1- and MC2R-embedded nanodiscs (CRHR1NDs and MC2RNDs) are immobilized on the floating electrode-based CNT-FET, and applied to detect CRH and ACTH, respectively.

Fig. 2. Characterization of CRHR1 and MC2R expressed in HEK-293 cells and E. coli (a)

Dose-dependent responses of CRHR1 expressed in HEK-293 cells to CRH $(n=3, * p<0.05$, $* * \mathrm{p}<0.01, * * * \mathrm{p}<0.001$ ). (b) Selectivity of CRHR1 expressed in HEK-293 cells after the addition of various hormones and neurotransmitters $(n=3, C R H$ : corticotropin-releasing hormone, ACTH: adrenocorticotropic hormone, DA: dopamine, EP: epinephrine, SE: serotonin). (c) Dose-dependent responses of MC2R expressed in HEK-293 cells to ACTH ( $n=3$, ${ }^{*} \mathrm{p}<0.05, * * \mathrm{p}<0.01, * * * \mathrm{p}<0.001$ ). (d) Selectivity of MC2R expressed in HEK-293 cells to various hormones and neurotransmitters $(n=3)$. (e) Western blot analysis of CRHR1 and MC2R expressed in E. coli using V5 Ab. The band indicates the molecular weight of CRHR1 (left image). The band corresponds to the molecular weight of MC2R (right image). (f) FE-SEM image of CRHR1NDs. The NDs had a discoidal shape, and their average size was approximately $20 \mathrm{~nm}$.

Fig. 3. Optical and electrical characterization of a bioelectronic sensor system (a) Optical image of fabricated bioelectronic sensor chip. (b) FE-SEM image of a bioelectronic sensor with a 
599 CNT channel and floating electrodes. The five floating electrodes were fabricated between the source and drain electrodes. The CNT channel was also observed under the floating electrodes. (c) I-V curves of a floating electrode-based bioelectronic system before and after the immobilization of NDs. The $\mathrm{dI} / \mathrm{dV}$ exhibited a linear behavior. When the NDs were immobilized on the floating electrodes, the $\mathrm{d} / \mathrm{dV}$ decreased as the resistance increased. However, the linearity was preserved after the immobilization of NDs. (d) Liquid gate profiles of a floating electrode-based bioelectronic sensor before and after the immobilization of NDs. The floating electrode-based CNT-FET showed a p-type profile. The p-type profile was maintained even after the immobilization of NDs on the floating electrodes.

Fig. 4. Electrical measurement of CRHR1ND- and MC2RND-based bioelectronic sensors. (a) Real-time responses of a CRHR1ND-based bioelectronic sensor to the various concentrations of CRH. As the concentration of CRH (1 fM to $1 \mathrm{pM}$ ) was introduced to the bioelectronic sensor, the conductance changed. (b) Dose-dependent responses of the CRHR1ND-based bioelectronic sensor to CRH. Real-time electrical measurements were repeated three times, and the mean value and standard deviation were calculated and plotted. Based on the graph, the equilibrium constant was estimated as $5.70 \times 10^{9} \mathrm{M}^{-1}$. (c) Selective response of the CRHR1NDbased bioelectronic sensor to various hormones and neurotransmitters $(1 \mathrm{nM})$. The conductance changed following the injection of $1 \mathrm{nM} \mathrm{CRH}$. However, the same concentration of SE, EP, and ACTH did not change the conductance in the sensor. (d) Real-time responses of a MC2RND-based bioelectronic sensor to various concentrations of ACTH. The introduction of different concentrations of ACTH (1 to $100 \mathrm{fM}$ ) changed the conductance. (e) Dose-dependent responses of the MC2RND-based bioelectronic sensor to ACTH. Real-time electrical measurements were repeated three times, and the mean value and standard deviation were 
623 calculated and plotted as each point. The estimated equilibrium constant was $5.47 \times 10^{10} \mathrm{M}^{-1}$. 624 (f) Selective response of the MC2RND-based bioelectronic sensor to various hormones and 625 neurotransmitters $(1 \mathrm{nM})$. The sensor exhibited a response only when $1 \mathrm{nM}$ ACTH was injected 626 into the system, and did not respond to SE, EP, or CRH.

627

628 Fig. 5. Dose-dependent responses of the CRHR1ND- and MC2RND-based bioelectronic 629 sensors to (a) CRH, and (b) ACTH in blood plasma respectively. The human blood plasma was pretreated and diluted at a 1:104 ratio. Different concentrations of the CRH and ACTH samples

631 from $10^{-18} \mathrm{M}$ to $10^{-5} \mathrm{M}$ were prepared by mixing it with the plasma. In the plasma environment, 632 the dose-response curves showed a similar slope to those in the buffer condition. Selectivity 633 of (c) CRHR1ND- and (d) MC2RND-based bioelectronic sensors to various hormones and 634 neurotransmitters in the diluted blood plasma, respectively $\left(\mathrm{n}=3,{ }^{*} \mathrm{p}<0.05,{ }^{*} \mathrm{p}<<0.01, * * * \mathrm{p}\right.$ $635<0.001)$ 
$637 \quad$ Fig. 1

638

639

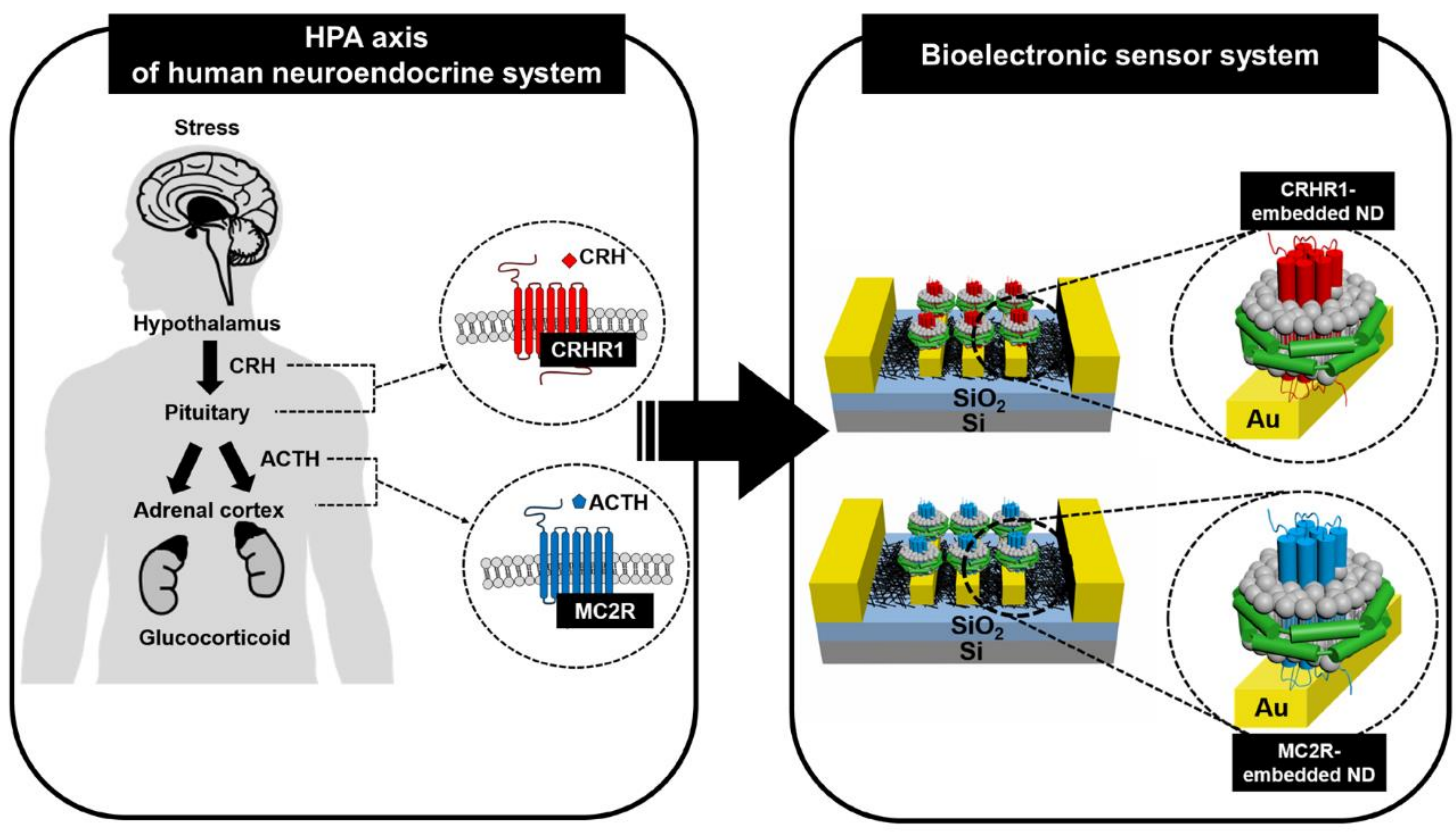

640

641 
Fig. 2

(a)

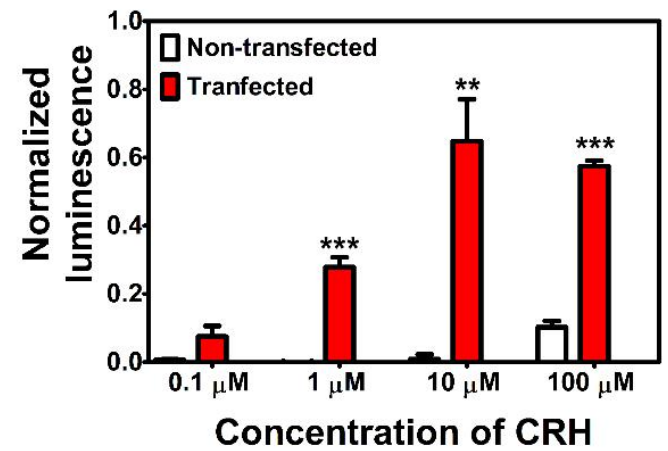

(c)

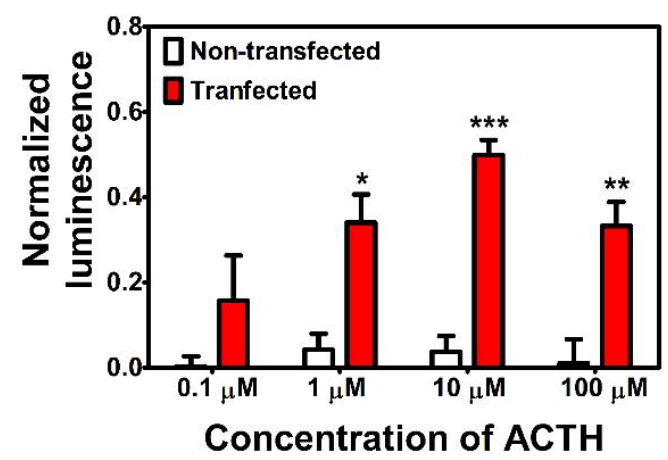

(e)

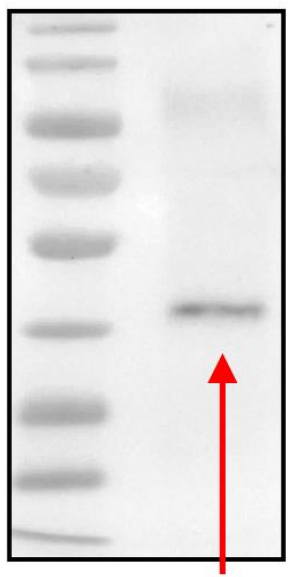

CRHR1

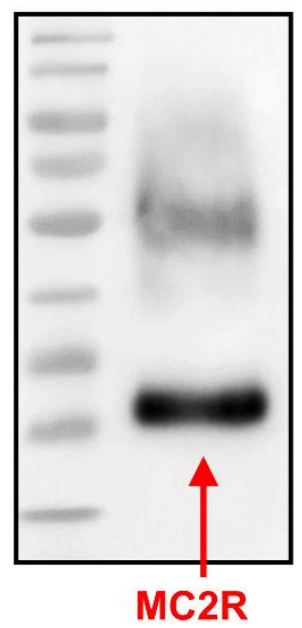

(b)

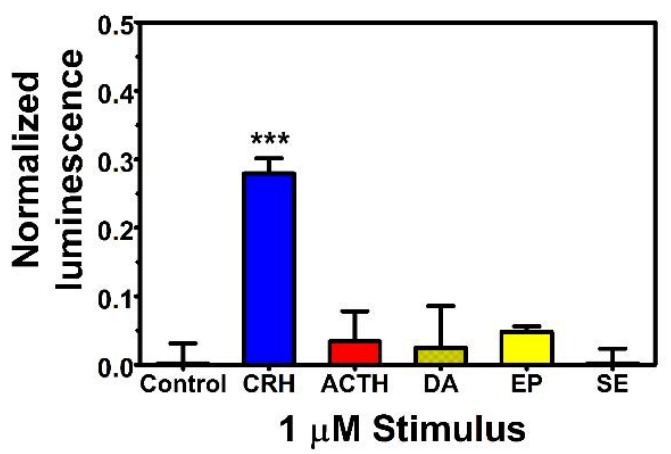

(d)

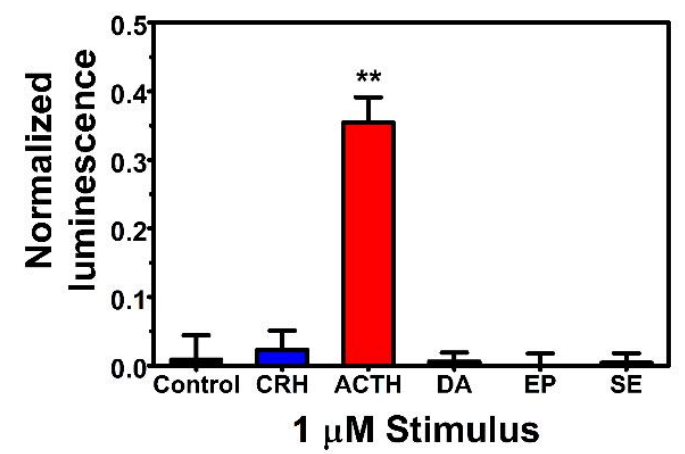

(f)

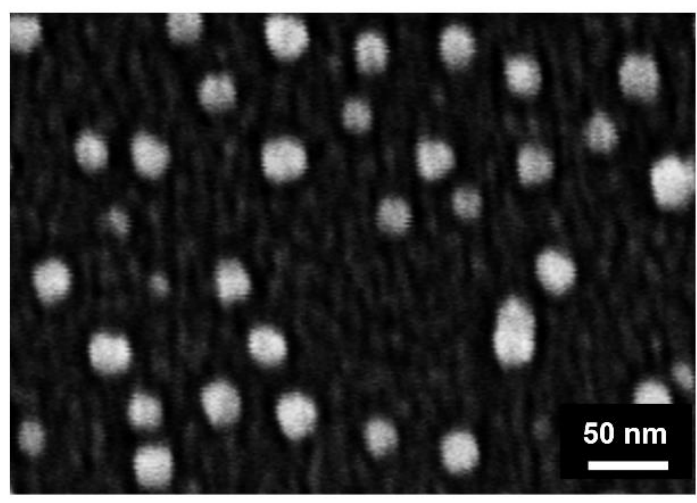


Fig. 3

648

(a)

(b)

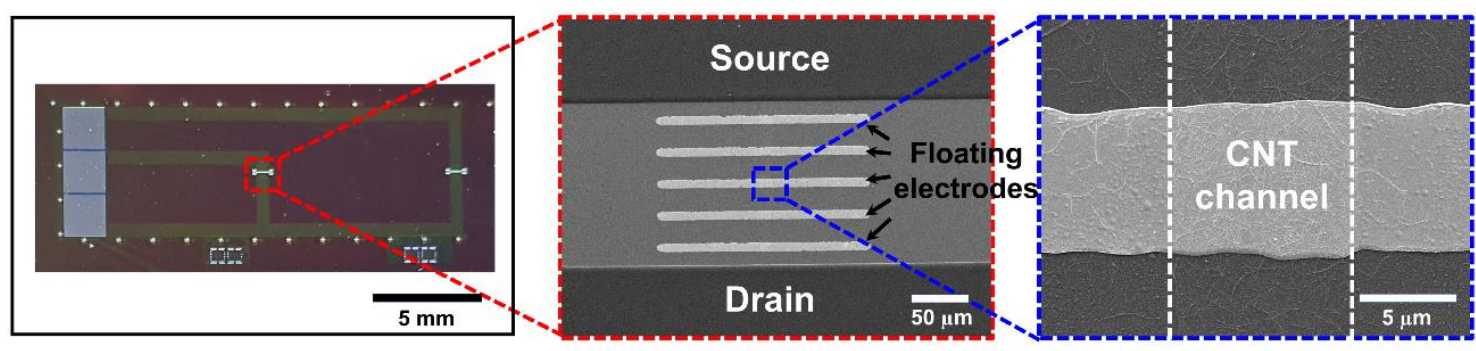

(c)

(d)
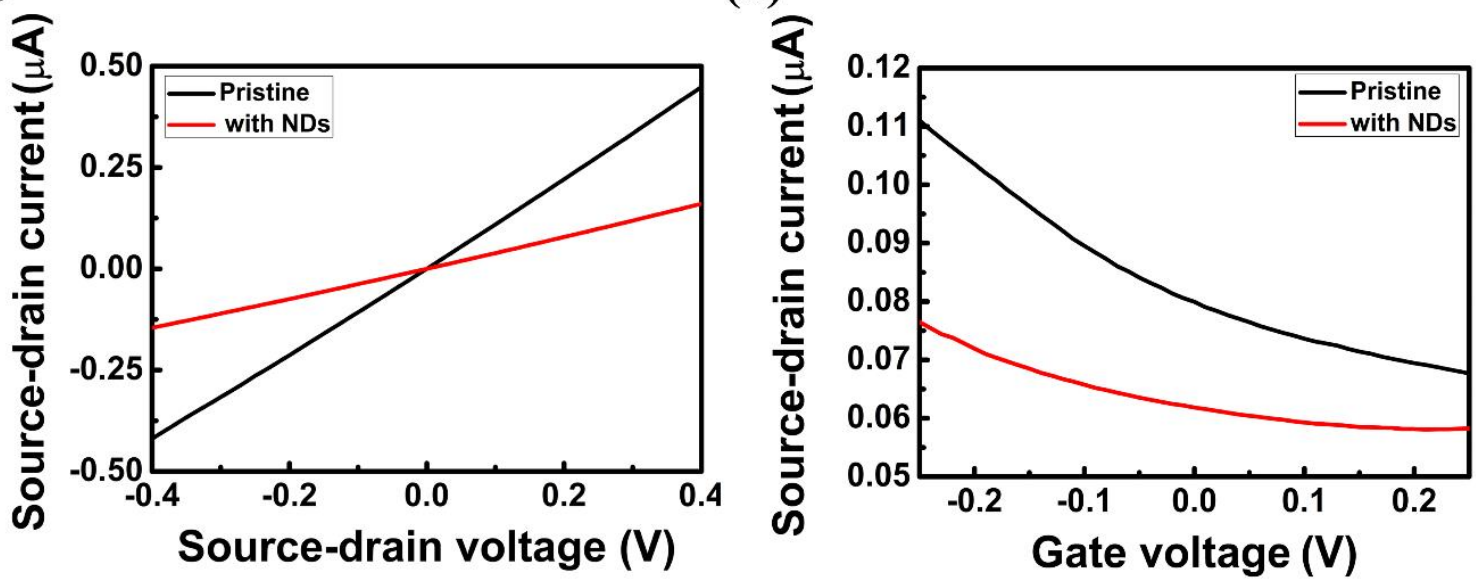
Fig. 4

653

(a)

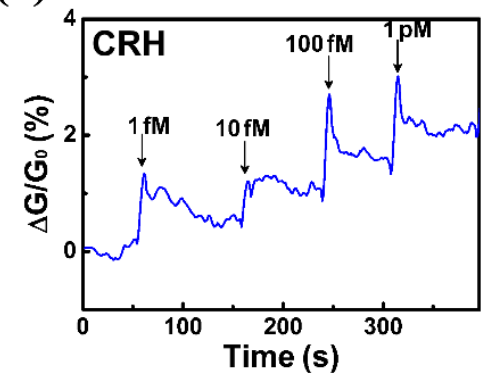

(d)

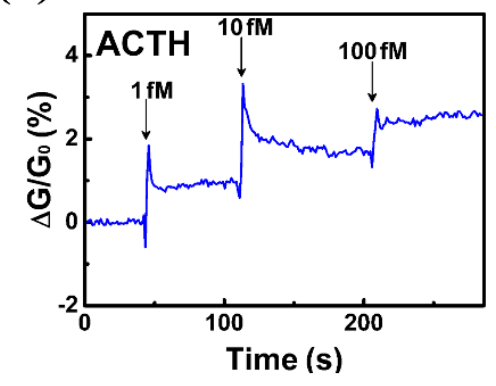

(b)

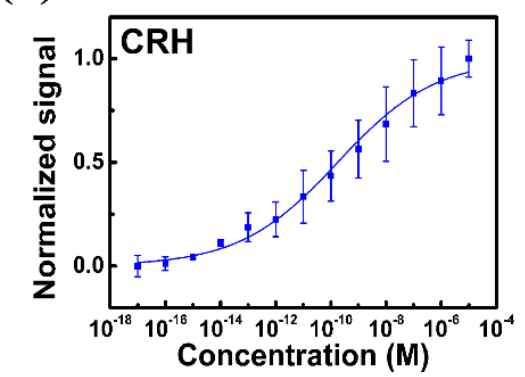

(e)

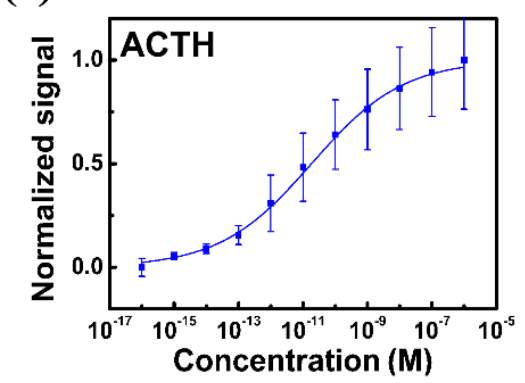

(c)

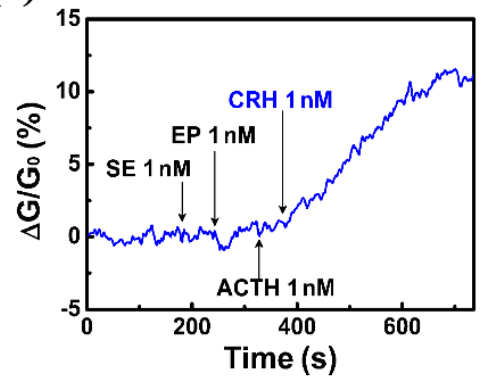

(f)

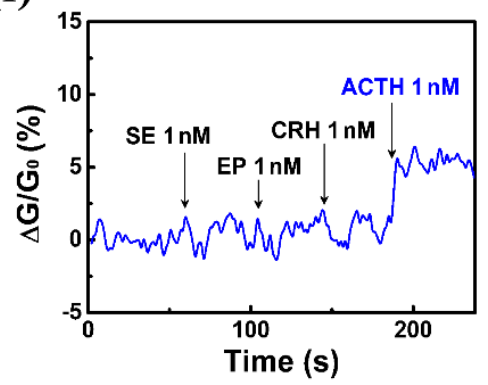

654

655

656 
Fig. 5

659

(a)

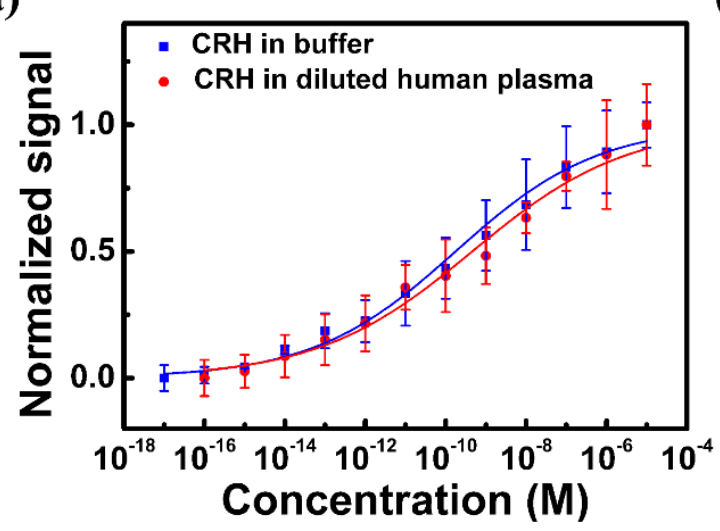

(c)

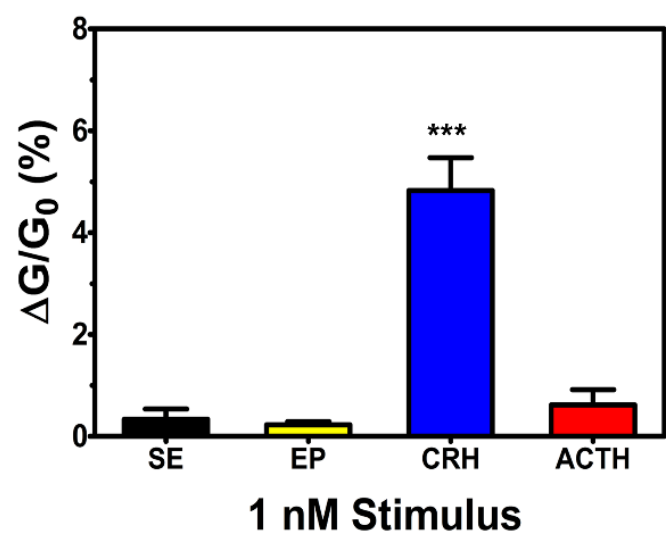

(b)

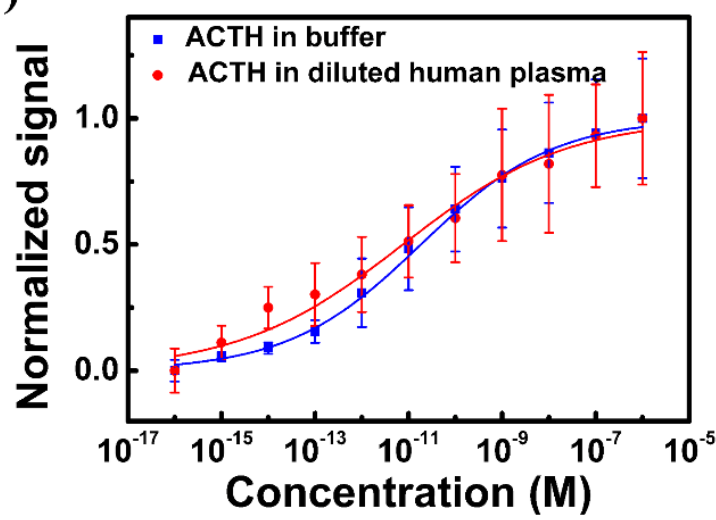

(d)

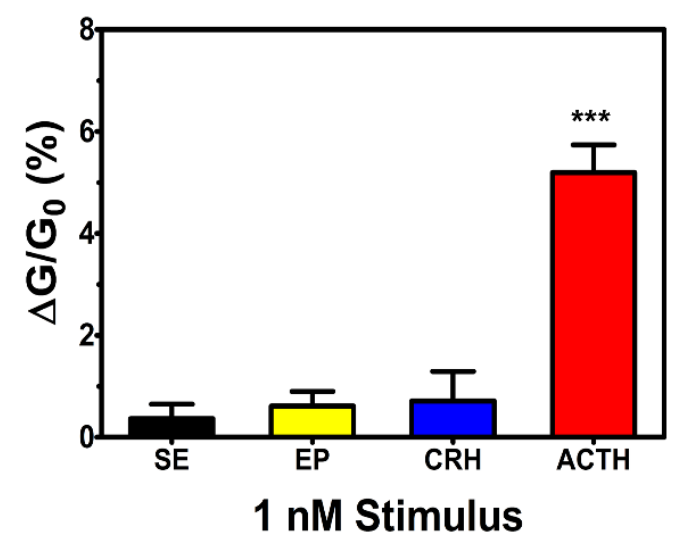

